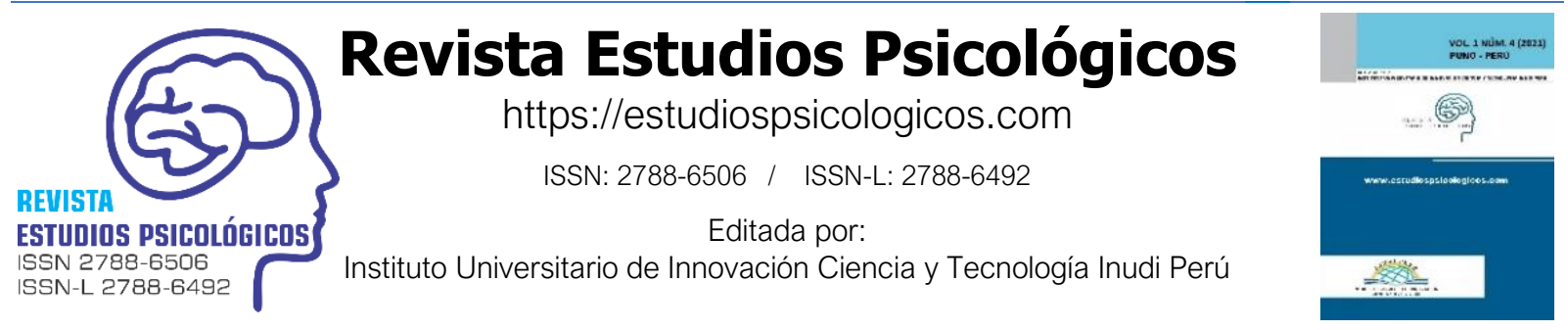

ARTÍCULO ORIGINAL

\title{
Efectos del humor, soledad y la ideación del divorcio en la satisfacción conyugal y vital de adultos peruanos casados
}

Effects of humor, Ioneliness and the ideation of divorce on the marital and vital satisfaction of married Peruvian adults

Efeitos do humor, da solidão e da ideação do divórcio na satisfação conjugal e vital de adultos peruanos casados

\author{
Cristian Ramos-Vera ${ }^{1}$ \\ Universidad Cesar Vallejo, Lima - Lima, Perú \\ (iD https://orcid.org/0000-0002-3417-5701
}

\section{Antonio Urbano \\ Universidad de Oviedo, España \\ https://orcid.org/0000-0001-6973-1125}

\author{
Antonio Serpa \\ Universidad Nacional Mayor de San Marcos, Lima - Lima, Perú \\ (iD https://orcid.org/0000-0002-7997-2464
}

DOI: https://doi.org/10.35622/j.rep.2022.01.003

Recibido 21/11/2021/ Aceptado 05/01/2022

PALABRAS CLAVE

divorcio, humor, parejas, satisfacción, soledad.

\section{KEYWORDS}

divorce, humor, couples, satisfaction, loneliness.
RESUMEN. Se tuvo como objetivo evaluar los efectos de la soledad, humor e ideación del divorcio en la satisfacción vital y conyugal en adultos peruanos. Este estudio cuantitativo de tipo predictivo se realizó mediante un modelo estructural. La muestra estuvo representada por 157 mujeres y 99 hombres que asistían a un centro de salud de Lima Metropolitana, con edades entre 18 y 89 años (Medad $=39.93)$. Los instrumentos de encuesta utilizados reportaron adecuadas evidencias de validez interna y correlacional, se reportó en total seis modelos estructurales, el último modelo incluyó los efectos de las variables mencionadas donde se presentaron mayores efectos negativos de la soledad ( $\beta=-.43, \beta=-.32)$; ideación de divorcio $(\beta=-.29$ y $\beta=-.22)$ y efectos positivos del humor $(\beta=.23, \beta=.24)$ en la satisfacción de pareja y personal respectivamente, todas las medidas de regresión fueron prácticamente significativas para la investigación psicológica. Los índices de bondad de ajuste refuerzan el modelo propuesto: $x 2 / g l=1.89, \mathrm{CFI}=.949, \mathrm{TLI}=.959, \mathrm{RMSEA}=.056$, SRMR=.069, que permiten confirmar de manera general la regresión múltiple latente y los objetivos propuestos de las variables de estudio.

ABSTRACT. The objective was to evaluate the effects of divorce's loneliness, humor, and creativity on life and marital satisfaction in Peruvian adults. This quantitative predictive study was carried out using a structural model. The sample was represented by 157 women and 99 men who attended a health center in Metropolitan Lima between 18 and 89 years (Average = 39.93). The survey instruments used reported adequate evidence of internal and correlational validity, a total of six

\footnotetext{
${ }^{1}$ Correspondencia: cristony_777@hotmail.com
} 
structural models were reported, the last model included the effects of the mentioned variables where there were greater negative effects of loneliness $(\beta=-.43, \beta=-.32)$; ideation of divorce ( $\beta$ $=-.29$ and $\beta=-.22)$ and positive effects of humor $(\beta=.23, \beta=.24)$ on partner and personal satisfaction respectively, all regression measures were practically significant for psychological research. The goodness of fit indices reinforces the proposed model: $\mathrm{x2} / \mathrm{gl}=1.89, \mathrm{CFI}=.949$, $\mathrm{TLI}=.959, \mathrm{RMSEA}=.056, \mathrm{SRMR}=.069$, which allow the general confirmation of the latent multiple regression and the objectives. proposed of the study variables.

PALAVRAS-CHAVE
divórcio, humor, casais,
satisfação, solidão.

RESUM0. 0 objetivo foi avaliar os efeitos da solidão, do humor e da ideação do divórcio na vida e na satisfação conjugal de adultos peruanos. Este estudo quantitativo preditivo foi realizado usando um modelo estrutural. A amostra foi representada por 157 mulheres e 99 homens que frequentavam um centro de saúde na região metropolitana de Lima, com idades entre 18 e 89 anos (Média = 39,93). Os instrumentos de pesquisa utilizados relataram evidências adequadas de validade interna e correlacional, um total de seis modelos estruturais foram relatados, o último modelo incluiu os efeitos das variáveis mencionadas onde houve maiores efeitos negativos da solidão ( $\beta=-.43, \beta=$ - .32); ideação de divórcio ( $\beta=-.29$ e $\beta=-.22$ ) e efeitos positivos do humor $(\beta=.23, \beta=.24$ ) na satisfação do parceiro e pessoal, respectivamente, todas as medidas de regressão foram praticamente significativas para a pesquisa psicológica. Os índices de adequação reforçam 0 modelo proposto: $\times 2$ / gl = 1,89, CFI = 0,949, TLI =0,959, RMSEA $=0,056$, SRMR $=0,069$, que permitem a confirmação geral da regressão múltipla latente e dos objetivos. propostas das variáveis de estudo.

\section{INTRODUCCIÓN}

Entre los múltiples subsistemas relacionales que constituyen el sistema familiar, la relación de pareja 0 subsistema conyugal es considerado el central (Davies et al., 2009; Fincham et al., 2018). Las relaciones de pareja se conforman cuando dos personas mantienen una relación sentimental, establecen entre ellas voluntad para convivir e interactuar, desean mantener una unión exclusiva, compartir un proyecto vital y manifiestan cierto nivel de compromiso. Todo esto las lleva a ser un escenario con numerosas experiencias emocionales (Granillo \& Sánchez, 2020; Minuchin, 1974).

Mantener una relación de pareja, en caso de que esta sea funcional, supone una fuente de apoyo emocional y social, la cual contribuye a la construcción de la identidad, a la mejora de la competencia social y, en definitiva, al bienestar personal (Hazan \& Diamond, 2000; Vidal et al., 2012). Sin embargo, una relación deteriorada afecta negativamente a la dinámica familiar, a la propia salud mental y física de la pareja e inclusive a su entorno (Irani et al., 2021; Lee et al., 2021; K. A. S. Wickrama \& 0’Neal, 2021).

La satisfacción con la relación de pareja supone un indicador fundamental para establecer el bienestar de sus miembros y de la propia relación (Urbano et al., 2018). La calidad o satisfacción en una relación de pareja es entendida como el grado en que los dos miembros de la pareja muestran intimidad, afecto y apoyo mutuo, aunque. También puede definirse como un estado emocional en el que nos sentimos complacidos con las interacciones, las experiencias y expectativas que tenemos con respecto a la relación de pareja (Collins et al., 2009; Ward et al., 2009). 
Dentro de las relaciones de pareja la satisfacción con la propia relación supone un elemento clave, siendo uno de los temas más abordados en la investigación sobre esta temática (M. García \& Romero, 2012). La satisfacción no se puede considerar un factor estático, pues se ve modificado conforme las relaciones avanzan en el tiempo. Desde su constitución, los dos miembros tienen expectativas diferentes que dependen, entre otras cuestiones, del género, la duración de la relación, tener hijos, con un impacto notable, lo que cada integrante de la pareja vivió y aprendió en su familia de origen (Garrido et al., 2008; Hernández et al., 2011; Urbano et al., 2019).

Como se ha comentado anteriormente, un correcto funcionamiento de la relación de pareja está vinculado con el propio bienestar y la satisfacción con la vida. De forma breve, la satisfacción vital puede entenderse como la discrepancia entre el estado actual en el que nos encontramos y el estado ideal que aspiramos alcanzar, según los objetivos vitales que se persiguen (Oliva et al., 2011, p. 108). Las dinámicas que tienen lugar en las relaciones de pareja (comunicación, conflicto, sexualidad, etc.) llevan asociadas una gran carga emocional que pueden condicionar la percepción y valoración que hacen las personas de su situación personal e, incluso, el propio estado conyugal (estar soltero, casado o divorciado). Esto puede ser un condicionante del nivel de felicidad manifestado (Alarcón, 2001; Bannon et al., 2020; Bastida et al., 2017).

La satisfacción vital es un constructo relacionado con tener pareja y es el indicador más estable del bienestar subjetivo, por lo que es fundamental su estudio (Cuadros et al., 2014; Emerson et al., 2017). Se debe considerar la relación de pareja al constatarse, por ejemplo, que las personas que tienen pareja son más felices que aquellas que no (Alzugaray \& García, 2015). Al hacer una diferenciación según el género, Ramírez y Lee (2012) al estudiar la satisfacción vital en mayores de 60 años encuentran diferencias entre hombres y mujeres, pero sin que estas sean significativas. Sin embargo, observan que los hombres solo presentan relación con la satisfacción vital al tomar en cuenta la variable ingresos económicos. Por su parte las mujeres suman otra serie de variables como cercanía y compañía de sus familias.

Vinculado con la satisfacción con la relación de pareja y la satisfacción con la propia vida, es interesante abordar el miedo a la separación de pareja. Cuando la unión entre la pareja empieza a decaer, surge la incomprensión, se reducen las expresiones de afecto y aumenta la desconfianza se genera un desgaste emocional que puede desencadenar a partir de la ideación de divorcio la ruptura de la relación conyugal (Dixon \& Wilhoit, 2019; Ward et al., 2009). Resulta fundamental profundizar en el estudio de posibles condicionantes, positivas y negativas, que puedan influir en la disposición de uno o ambos miembros de la pareja a dar por finalizado el vínculo.

Una ruptura de pareja puede conllevar un incremento de los niveles de estrés y suele verse como una de las experiencias más dolorosas a nivel vital que se pueden experimentar, llegan a afectar incluso la salud física y mental de la pareja (Barajas \& Cruz, 2017; F. García \& llabaca, 2013). De forma paralela, la afección es también 
para su entorno, al poder verse deteriorado gravemente el sistema familiar, especialmente cuando cuentan con descendencia (Fariña et al., 2017).

Con respecto a posibles condicionantes que contribuyan a proteger o deteriorar la relación, en esta investigación se busca profundizar en el estudio del humor como variable protectora y de la soledad como amenaza. Respecto al humor, ha quedado demostrado que ayuda a fortalecer la dinámica conyugal, que influye desde el momento de la propia elección de la pareja (Caird \& Martin, 2014; Maté \& Acarín, 2011; Saroglou et al., 2010; Valdez et al., 2005; Zicavo \& Vera, 2011). Situación que incluso, contribuye a predecir la satisfacción con la relación (Bippus et al., 2011; Martin \& Ford, 2018). En esta línea Flórez y Sánchez (2019) al estudiar la relación entre optimismo, resiliencia y sentido del humor en personas con pareja, destacan que existe tal relación y que es positiva respecto a la salud, especialmente referente a vitalidad.

En cuanto al sentimiento de soledad, se debe tener en cuenta las repercusiones negativas que puede generar en las personas y, vinculado a la relación de pareja, mantener o no este tipo de relaciones permite explicar el sentimiento de soledad social y emocional (Dykstra \& Fokkema, 2007; Flora \& Segrin, 2000; Jong et al., 2009). Asimismo, la soledad también estaría relacionada con la satisfacción con las relaciones personales, el humor y la satisfacción con la vida (Fitts et al., 2009; MacDonald et al., 2020; Mellor et al., 2008). Este estudio plantea evaluar los efectos que pueden tener las variables de soledad, ideación de divorcio y el humor de afrontamiento en la satisfacción vital y de pareja en peruanos.

\section{MÉTODO Y MATERIALES}

El presente estudio tuvo en cuenta a 256 adultos peruanos casados que acuden a un establecimiento de salud, de los cuales 157 (61.3\%) son mujeres y 99 (38.7\%) varones, cuyas edades están comprendidas entre 18 y 89 años ( $M E=39.93, \mathrm{DS}=13.23$ ). Se utilizó una calculadora de tamaño a priori para hallar el tamaño de muestra necesario para el análisis factorial confirmatorio (AFC) mediante el modelamiento de ecuaciones estructurales. Dado el número de variables observadas y latentes en el modelo, el tamaño mínimo del efecto previsto de .20 (para modelos predictivos), un nivel de probabilidad de .05 y potencia estadística de .85 (Ramos et al., 2021; Ramos \& Sáenz, 2021). Se estimó un número mínimo de 255 participantes.

\section{Instrumento}

Se utilizó la escala de evaluación de relaciones (Relationship Assessment Scale - RAS), versión en español, con siete reactivos para evaluar la satisfacción de la relación en pareja, compuesta por cinco opciones de respuesta (Hendrick, 1988; Moral, 2008). En el presente estudio la fiabilidad general es de .72. 
Escala de satisfacción con la vida (SWLS),la cual está conformada por cinco ítems y tiene como objetivo evaluar el juicio general de las personas acerca de la satisfacción con su vida (Diener et al., 2010). Para este estudio se usó la versión peruana de la SWLS de De la Cruz et al. (2018) con cinco opciones de respuesta. En el presente estudio, la fiabilidad de la SWLS, evaluada por el coeficiente alfa de Cronbach, fue de .79.

Escala de soledad (RULS-6) en su versión peruana, con seis ítems de respuesta politómica de cinco opciones, donde 1 significa totalmente en desacuerdo y 5 significa totalmente de acuerdo (Andia \& Castillo, 2021; Wongpakaran et al., 2020). Este escalamiento de cinco puntos fue elegido debido a que brinda más información acerca de las características evaluadas y estabilidad de las respuestas (Lozano et al., 2008; Ramos et al., 2021). La fiabilidad total en el presente estudio fue satisfactoria, con alfa de Cronbach igual a .75.

Escala de humor aplicado al afrontamiento (CHS), la cual tiene un ítem único: "Generalmente busco algo gracioso que decir cuando estoy en situaciones tensas" que evalúa la medida del sujeto en el uso del humor para hacer frente a la presión (Lin et al., 2018; Martin \& Lefcourt, 1983). Se pidió a los encuestados que respondieran cómo estaba de acuerdo o en desacuerdo con este ítem en una escala tipo Likert de 5 puntos (1 significa totalmente en desacuerdo y 5 significa totalmente de acuerdo).

\section{Procedimientos}

Los participantes completaron, la versión en español del SWLS y de la RAS, proporcionando también información acerca de su edad, sexo, convivencia. En cuanto a las covariables se tuvo en cuenta, la versión peruana del RULS-6, la pregunta única del CHS y la ideación de divorcio o separación: “¿Has pensado alguna vez en separarte de tu pareja?" (Hawkins et al., 2017). La administración de los instrumentos se realizó a cada persona de manera individual, contando con la autorización de las autoridades del centro de salud y teniendo en consideración las normativas éticas y la firma de consentimiento informado de cada uno de los participantes.

\section{Análisis de datos}

Se tuvo en cuenta los modelos de medición de las dos variables latentes (satisfacción de vida y de pareja) mediante AFC. Luego se evaluó el modelo estructural de la relación de ambas variables. Para analizar el efecto de las covariables mencionadas, a través de una técnica de modelado de ecuaciones estructurales (SEM). De forma específica, se utilizó un modelo predictivo referido como MIMIC (Múltiple Indicador y Causas Múltiples), que permite probar el impacto de un grupo de covariables en un modelo de medición que incluye variables latentes (Schumacker \& Lomax, 1996).

El uso de un modelo MIMIC es más adecuado que análisis de regresión para este tipo de investigación porque considera el efecto de cada elemento por separado. Por lo tanto, también estiman los errores de medición de 
cada componente de forma independiente. Por último, los modelos SEM, que incluyen los modelos MIMIC, son especialmente diseñados para probar construcciones que no son directamente observables, pero se miden mediante una serie de indicadores. Ya que este tipo de construcción es muy frecuente en la literatura psicológica. El uso de los modelos MIMIC es muy apropiado en este estudio, los cuales se han utilizado en estudios peruanos recientes (Baños \& Ramos, 2020; Bollen \& Bauldry, 2011; Ramos \& Sáenz, 2021).

Para brindar evidencias de los modelos estructurales SEM se realizó a través del programa estadístico Jasp 12.2 (Jasp Team, 2020). Se empleó el método de ULS, dado el criterio de la distribución multivariada en datos ordinales, idóneo para modelos predictivos; considerando buen ajuste a valores estimados por debajo o iguales a tres (Beauducel \& Herzberg, 2006; Gana \& Broc, 2019; Schumacker \& Lomax, 1996). Respecto a los índices de ajuste incremental valores CFI y TLI ( $\geq .90)$, SRMR ( $\leq .08)$, RMSEA ( $\leq .08)$ son considerados óptimos (Hu \& Bentler, 1999). Estos parámetros son utilizados en el espectro de la psicología cuantiaba (Ayandele et al., 2021; Núñez et al., 2021; Olapegba et al., 2021). Por último, para verificar las evidencias de la confiabilidad de los instrumentos se analizó a través del método de consistencia interna con los coeficientes $a \geq .70$ (Hair et al., 2010).

\section{RESULTADOS}

En la tabla 1 se muestra un resumen de los datos descriptivos de las variables de estudio. Presentan valores aceptables de distribución de normalidad univariada y multivariada. Las estructuras factoriales mediante AFC presentan índices de medición aceptables para las variables latentes de estudio.

Tabla 1

Datos descriptivos de las variables de estudio

\begin{tabular}{lllll}
\hline Variables & $M E$ & $D E$ & $g 1$ & $g 2$ \\
\hline RULS & 2.309 & 0.72 & 0.84 & 0.08 \\
SWLS & 3.55 & 0.23 & -0.38 & -0.65 \\
CHS & 2.84 & 1.30 & 0.16 & -1.04 \\
RAS & 3.62 & 0.11 & -0.15 & -0.36 \\
IS-D & 1.77 & 0.82 & 0.43 & 0.30 \\
\hline
\end{tabular}

Notas: ME: media, DE: desviación estándar, g1: asimetría, g2: curtosis RULS: soledad, SWLS: satisfacción de la vida, CHS: humor como afrontamiento, RAS: satisfacción de pareja, IS-D ideación de separación 0 divorcio.

En la tabla 2, se presentaron cargas factoriales mayores a .40 (Sellin \& Keeves, 1997). Los puntajes de confiabilidad de a fueron mayores a 70 . 
Tabla 2

Cargas factoriales del SWLS y RAS

\begin{tabular}{ccc} 
SWLS & $\lambda$ & $a$ \\
\hline SV1 & .704 & \\
SV2 & .678 & .794 \\
SV3 & .812 & \\
SV4 & .741 & \\
SV5 & .613 & \\
\hline RAS & 1 & \\
SP1 & .784 & .718 \\
SP2 & .508 & \\
SP3 & .565 & \\
SP4 & .600 & \\
SP5 & .649 & \\
SP6 & .428 & \\
SP7 & .530 &
\end{tabular}

Con respecto al modelo estructural de la relación de ambas variables también se presentó adecuados índices de ajuste SEM. Posteriormente se añadió seis análisis de ruta de las tres covariables hacia las dos variables latentes, aunque el ajuste disminuyó ligeramente, se mantuvo dentro de los rangos aceptables evidenciando la parsimonia del modelo MIMIC (X2/gI = 1.89); (SRMR =.069), (RMSEA=.056) y (CFI = .95; TLI=.96).

Tabla 3

Coeficientes estandarizados para covariables del modelo MIMIC

\begin{tabular}{llll}
\hline Covariables & RULS & ID & CHS \\
\hline Satisfacción de pareja & $-0.43^{\star *}$ & $-0.29^{* *}$ & $0.23^{* *}$ \\
Satisfacción vital & $-0.32^{\star *}$ & $-0.22^{* *}$ & $0.24^{* *}$ \\
\hline
\end{tabular}

Nota: RULS: soledad, ID: ideación de divorcio-separación, CHS: humor como afrontamiento, ${ }^{*} p<.05 ;{ }^{* *} p<.01$

Los resultados de los seis coeficientes estandarizados añadidos se muestran en la tabla 4. Las covariables consideradas en el modelo general MIMIC (M2) fueron la ideación de divorcio, la soledad y el afrontamiento como humor. La ideación de divorcio y la soledad predijeron de manera negativa en la satisfacción de vida y 
pareja. Mientras que el humor como afrontamiento tienen un impacto positivo significativo con la satisfacción en la persona y en las relaciones.

Tabla 4

Índice de Bondad de ajuste de los modelos de estudio $(\mathrm{n}=256)$

\begin{tabular}{lccccc}
\hline Modelo & $X^{2} / g l$ & CFI & TLI & SRMR & RMSEA \\
\hline RULS-6 & 1.66 & 0.995 & 0.992 & 0.049 & 0.051 \\
RAS & 1.06 & 0.998 & 0.997 & 0.048 & 0.016 \\
SWLS & 1.16 & 0.999 & 0.999 & 0.026 & 0.025 \\
M1 & 1.83 & 0.979 & 0.974 & 0.065 & 0.057 \\
M2 & 1.89 & 0.949 & 0.959 & 0.069 & 0.056 \\
\hline
\end{tabular}

Nota: $x^{2} / \mathrm{gl}$ : chi-cuadrado sobre grado de libertad, CFI: índice de ajuste comparativo; TLI: índice

Tucker- Lewis; SRMR: raíz residual estandarizada cuadrática media; RMSEA: raíz cuadrada del error medio cuadrático; M1: modelo estructural de correlación, M2: M1 con efectos de covariables

\section{DISCUSIONES}

Los resultados evidencian la relación entre satisfacción de pareja y satisfacción vital de acorde a la literatura psicológica (Adamczyk, 2019; Czyżowska et al., 2020; Margelisch et al., 2017; Rodríguez et al., 2019). La investigación agrega más evidencia confirmatoria, dado el modelo estructural propuesto de los efectos del humor como afrontamiento, la soledad e ideación de divorcio en las variables mencionadas.

A pesar de contar mayormente con una serie de estudios en adultos que han evidenciado una asociación negativa de la soledad en la satisfacción vital, este vínculo también puede verse reflejado en las relaciones de pareja, debido a que no siempre se proporcionan los beneficios esperados a los conyugues (Cao \& Lu, 2021; Huo \& Kong, 2014; Lawal \& Okereke, 2021; Marilaf et al., 2017; Tian et al., 2018; Turan et al., 2020). Por lo tanto, se ha podido identificar como un predictor de la insatisfacción marital a la soledad (Mund et al., 2020). Es decir, que la soledad es un factor de riesgo en las relaciones maritales debido a las amplias repercusiones negativas intra e inter personales que afectan a los adultos (Holt et al., 2015; Saporta et al., 2021; K. Wickrama et al., 2020). Un estudio longitudinal de Mund y Johnson (2021) determinó que una mayor soledad predijo un declive en la satisfacción personal y de pareja. Se considera que las personas más solitarias prestan mayor 
atención a posibles señales de rechazo o exclusión social y presentan dificultad en el restablecimiento de la confianza (Knoke et al., 2010).

La falta de atribución positiva hacia la pareja en la relación está vinculado a una menor sensación de intimidad, cercanía y afecto emocional que puede conllevar a que uno de ellos se sienta solo. Esto genera una serie de sentimientos y pensamientos asociados al término de la relación o refuercen una incertidumbre al compromiso de pareja (Crabtree et al., 2018; Fincham et al., 2018; Hoy et al., 2022). Esto puede afianzar la ideación de divorcio, pues en la actualidad en una relación que no es plenamente satisfactoria, es común que surja la pregunta sobre la posibilidad de la disolución marital, lo cual pone en cuestionamiento la situación conyugal, siendo conocido como una de las principales actitudes al divorcio (Baxter, 2010; Crabtree et al., 2018; Dixon \& Wilhoit, 2019; Doherty et al., 2021; Doherty \& Harris, 2017; Galovan et al., 2021; Goodboy et al., 2020; Hawkins et al., 2017). Incluso, esto puede vincularse a la sensación de soledad, donde el individuo no se siente correspondido íntima y emocionalmente por su pareja (Knoke et al., 2010; Margelisch et al., 2017). Lo que permite a la persona preferir estar sola y considerar la decisión de separarse.

Por otro lado, las parejas que presentan humor para afrontar el estrés gozan de bienestar personal y marital, lo cual favorece el manejo de la tensión en la relación y permite la regulación de las emociones de ambos conyugues (Celso et al., 2003; Y. Flórez \& Sánchez, 2021; Nezlek \& Derks, 2001; Vela et al., 2013). Por consiguiente, el humor puede ser considerado como un factor a tener en cuenta para las terapias de pareja ante los problemas de incertidumbre y soledad, debido al sentimiento de déficit en la calidad de la relación conyugal (MacDonald et al., 2020; Martin \& Ford, 2018).

Se debe tener en cuenta que este estudio se centra en diversos contenidos temáticos relacionados a la investigación de las relaciones de pareja en participantes sudamericanos. Siendo el primer estudio hasta donde se incluye la medida de ideación de divorcio en hispanohablantes considerado el tema de mayor interés de la satisfacción de pareja. Una revisión sistemática reciente revela que durante las últimas dos décadas la mayoría de investigaciones sobre parejas en Latinoamérica fueron realizadas en los países de México y España, donde se tuvo una tendencia a favor del uso de una sola temática, respecto a la investigación en las relaciones. Aproximadamente el 10\% de los estudios incluyeron más de dos variables (Urbano et al., 2021). Además, señalaron únicamente un estudio peruano realizado por Zicavo y Vera (2011). Por tanto, el presente artículo es pionero en el Perú dado que incluyó variables personales como la soledad y el humor que predicen la satisfacción relacional y vital.

Las limitaciones del estudio pueden residir en el diseño transversal del estudio dado que se realizó en un momento dado. A su vez, solo se tuvo en cuenta personas de un centro de salud de un solo distrito de la capital 
peruana, sin tomar en cuenta la inclusión de evaluados de diferentes niveles socioeconómicos. Por lo tanto, para futuros estudios se recomienda un análisis más detallado de diferentes características sociodemográficas, y la inclusión de otras variables contextuales vinculados a la relación de pareja. En conclusión, el presente estudio confirma el modelo estructural de los efectos positivos del humor en una mejor satisfacción de la persona y en su relación, mientras que la ideación del divorcio y la soledad son factores que afectan los niveles de satisfacción.

\section{CONCLUSIONES}

En conclusión, se demostraron adecuadas evidencias de confiabilidad y validez de los instrumentos utilizados que permitieron realizar el análisis estructural propuesto. Se consideró la relación latente entre la satisfacción de la vida de las parejas y de su relación. Así como el efecto de covariables de naturaleza personal y conyugal, donde destacó mayores efectos negativos de la soledad y de la ideación suicida, mientras el humor como afrontamiento predijo ambas medidas de satisfacción.

Vale la pena mencionar el análisis estadístico utilizado para confirmar los objetivos planteados y permiten una mayor aproximación de aspectos poco explorados en la investigación psicológica de las relaciones de pareja, especialmente en el Perú.

El presente estudio permite conocer sobre el aspecto de la satisfacción a nivel personal y sobre la relación. Los resultados pueden ser de interés para futuros estudios que consideren diferentes grupos sociodemográficos de parejas. Asimismo, el estudio de otras variables más allá de las más estudiadas como la comunicación de pareja que permitan comprender los mecanismos que median las relaciones entre tales variables de mayor interés a la población en cuestión.

\section{Conflicto de intereses / Competing interests:}

Los autores declaran que no incurre en conflictos de intereses.

\section{Rol de los autores / Authors Roles:}

Cristian Ramos-Vera: conceptualización, curación de datos, análisis formal, adquisición de fondos, investigación, metodología, administración del proyecto, recursos, software, supervisión, validación, visualización, escritura preparación del borrador original, escritura - revisar \& amp; edición.

Antonio Serpa: conceptualización, análisis formal, investigación, metodología, administración del proyecto, recursos, software, supervisión, validación, visualización, escritura - preparación del borrador original, escritura - revisar \& amp; edición.

Antonio Urbano: conceptualización, análisis formal, investigación, metodología, administración del proyecto, recursos, software, supervisión, validación, visualización, escritura - preparación del borrador original, escritura revisar \& amp; edición. 


\section{Fuentes de financiamiento / Funding:}

Los autores declaran que no recibió un fondo específico para esta investigación.

\section{Aspectos éticos / legales; Ethics / legals:}

Los autores declaran no haber incurrido en aspectos antiéticos, ni haber omitido aspectos legales en la realización de la investigación.

\section{REFERENCIAS}

Adamczyk, K. (2019). Incremental Validity of the Satisfaction with Relationship Status Scale in Predicting Young Adults' Well-Being. What Does It Tell Us? Current Psychology, 38(3), 865-872. https://doi.org/10.1007/s12144-017-9666-9

Alarcón, R. (2001). Relaciones entre felicidad, género, edad y estado conyugal. Revista de Psicología, 19(1), 27-46. https://doi.org/10.18800/psico.200101.002

Alzugaray, C., \& García, F. (2015). Relaciones de pareja y bienestar. https://www.researchgate.net/publication/294891178_Relaciones_de_pareja_y_bienestar_psicologico

Andia, M., \& Castillo, R. (2021). Estructura factorial de la escala "Falta de personal" en trabajadores de una empresa retail peruana. Revista de Investigación en Psicología, 24(1), 91-100. https://doi.org/10.15381/rinvp.v24i1.20615

Ayandele, 0., Ramos, C., Iorfa, S. K., Chovwen, C., \& Olapegba, P. (2021). Exploring the Complex Pathways between the Fear of COVID-19 and Preventive Health Behavior among Nigerians: Mediation and Moderation Analyses. The American Journal of Tropical Medicine and Hygiene, 105(3), 701-707. https://doi.org/10.4269/ajtmh.20-0994

Bannon, S., Taggart, T., Kehoe, C., \& O'Leary, D. (2020). Collaborative communication efficiency is linked to relationship satisfaction in dating couples. Personal Relationships, 27(2), 385-400. https://doi.org/10.1111/pere.12319

Baños, J., \& Ramos, C. (2020). Validez e invarianza según sexo y edad de la Escala Paykel de Ideación Suicida en adolescentes peruanos. Interacciones: Revista de Avances en Psicología, 6(1), e225. https://doi.org/10.24016/2020.v6n1.225

Barajas, M., \& Cruz, C. (2017). Ruptura de la pareja en jóvenes: factores relacionados con su impacto. Enseñanza e Investigación en Psicología, 22(3), 342-352. https://www.redalyc.org/articulo.oa?id=29255775008

Bastida, R., Valdez, J., Valor, I., González, N., \& Rivera-Aragón, S. (2017). Satisfacción marital y estado civil como factores protectores de la depresión y ansiedad. Revista argentina de clínica psicológica, 26(1), 95102. http://ri.uaemex.mx/handle/20.500.11799/79188 
Baxter, L. (2010). The Dialogue of Marriage. Journal of Family Theory \& Review, 2(4), 370-387. https://doi.org/10.1111/j.1756-2589.2010.00067.x

Beauducel, A., \& Herzberg, P. (2006). On the performance of maximum likelihood versus means and variance adjusted weighted least squares estimation in CFA. Structural Equation Modeling: A Multidisciplinary Journal, 13(2), 186-203. https://doi.org/10.1207/s15328007sem1302_2

Bippus, A., Young, S., \& Dunbar, N. (2011). Humor in conflict discussions: Comparing partners' perceptions. Humor - International Journal of Humor Research, 24(3). https://doi.org/10.1515/humr.2011.018

Bollen, K., \& Bauldry, S. (2011). Three Cs in measurement models: Causal indicators, composite indicators, and covariates. Psychological Methods, 16(3), 265-284. https://doi.org/10.1037/a0024448

Caird, S., \& Martin, R. (2014). Relationship-focused humor styles and relationship satisfaction in dating couples: A repeated-measures design. HUMOR, 27(2). https://doi.org/10.1515/humor-2014-0015

Cao, Q., \& Lu, B. (2021). Mediating and moderating effects of loneliness between social support and life satisfaction among empty nesters in China. Current Psychology, 40(2), 973-982. https://doi.org/10.1007/s12144-018-0019-0

Celso, B., Ebener, D., \& Burkhead, E. (2003). Humor coping, health status, and life satisfaction among older adults residing in assisted living facilities. Aging \& Mental Health, 7(6), 438-445. https://doi.org/10.1080/13607860310001594691

Collins, A., Welsh, D., \& Furman, W. (2009). Adolescent Romantic Relationships. Annual Review of Psychology, 60(1), 631-652. https://doi.org/10.1146/annurev.psych.60.110707.163459

Crabtree, S., Harris, S., Bell, N., Allen, S., \& Roberts, K. (2018). The Roles of Love and Happiness in Divorce Decision Making. Journal of Divorce \& Remarriage, 59(8), 601-615. https://doi.org/10.1080/10502556.2018.1466254

Cuadros, J., Pérez, G., López, M., Cuadros, Á., \& Fernández, A. (2014). Satisfacción vital y factores sociodemográficos en mujeres de mediana edad. Enfermería Clínica, 24(6), 315-322. https://doi.org/10.1016/j.enfcli.2014.07.003

Czyżowska, D., Gurba, E., Czyżowska, N., \& Kalus, A. (2020). Intimate relationship and its significance for eudaimonic well-being in young adults. Health Psychology Report, 8(2), 155-166. https://doi.org/10.5114/hpr.2020.93768

Davies, P., Sturge, M., Woitach, M., \& Cummings, M. (2009). A process analysis of the transmission of distress 
from interparental conflict to parenting: Adult relationship security as an explanatory mechanism. Developmental Psychology, 45(6), 1761-1773. https://doi.org/10.1037/a0016426

De la Cruz, G., Lozano, F., Cantuarias, A., \& Ibarra, L. (2018). Validación de la escala satisfacción con la vida en trabajadores peruanos. Liberabit: Revista Peruana de Psicología, 24(2), 249-264. https://doi.org/10.24265/liberabit.2018.v24n2.06

Diener, E., Kahneman, D., Tov, W., \& Arora, R. (2010). Income's Association with Judgments of Life Versus Feelings. En International Differences in Well-Being (pp. 3-15). Oxford University Press. https://doi.org/10.1093/acprof:oso/9780199732739.003.0001

Dixon, L., \& Wilhoit, S. (2019). Divorced Families. En Encyclopedia of Couple and Family Therapy (pp. 787790). Springer International Publishing. https://doi.org/10.1007/978-3-319-49425-8_484

Doherty, W., \& Harris, S. (2017). Helping couples on the brink of divorce: Discernment counseling for troubled relationships.

Doherty, W., Kalantar, S., \& Tarsafi, M. (2021). Divorce ambivalence and reasons for divorce in Iran. Family Process, 60(1), 159-168. https://doi.org/10.1111/famp.12539

Dykstra, P., \& Fokkema, T. (2007). Social and emotional loneliness among divorced and married men and women: comparing the deficit and cognitive perspectives. Basic and Applied Social Psychology, 29(1), 1 12. https://doi.org/10.1080/01973530701330843

Emerson, S., Guhn, M., \& Gadermann, A. (2017). Measurement invariance of the satisfaction with life scale: reviewing three decades of research. Quality of Life Research, 26(9), 2251-2264. https://doi.org/10.1007/s11136-017-1552-2

Fariña, F., Pérez, V., Vázquez, M., \& Seijo, D. (2017). Clima familiar y coparentalidad en familias con ruptura de pareja. Revista de Estudios e Investigación en Psicología y Educación, 295-298. https://doi.org/10.17979/reipe.2017.0.05.2782

Fincham, F., Rogge, R., \& Beach, S. (2018). Relationship Satisfaction. En The Cambridge Handbook of Personal Relationships (pp. 422-436). Cambridge University Press. https://doi.org/10.1017/9781316417867.033

Fitts, S., Sebby, R., \& Zlokovich, M. (2009). Humor styles as mediators of the shyness-loneliness relationship. $\begin{array}{lllll}\text { Southeast } & \text { Missouri } & \text { State } & \text { University, } & \text { 11(2), }\end{array}$ https://go.gale.com/ps/i.do?id=GALE\%7CA200919506\&sid=googleScholar\&v=2.1\&it=r\&linkaccess=abs \&issn=15277143\&p=HRCA\&sw=w\&userGroupName=anon 8b2150eb 
Flora, J., \& Segrin, C. (2000). Relationship development in dating couples: implications for relational satisfaction and Ioneliness. Journal of Social and Personal Relationships, 17(6), 811-825. https://doi.org/10.1177/0265407500176006

Flórez, N., \& Sánchez, R. (2019). Optimismo, resiliencia, sentido del humor y la salud de personas con pareja. Pensando Psicología, 15(26), 1-24. https://doi.org/10.16925/2382-3984.2019.02.03

Flórez, Y., \& Sánchez, R. (2021). Satisfacción con la relación: contribuciones del humor, optimismo y resiliencia. $\begin{array}{llll}\text { Revista Electrónica de Psicología } & \text { Iztacala, }\end{array}$ http://www.revistas.unam.mx/index.php/repi/article/view/79788

Galovan, A., Hawkins, A., Harris, S., \& Simpson, D. (2021). What are they doing? A national survey of helpseeking and relationship-repair behavior of individuals who are thinking about divorce. Journal of Marital and Family Therapy, jmft.12480. https://doi.org/10.1111/jmft.12480

Gana, K., \& Broc, G. (2019). Structural equation modeling with lavaan, London. Wiley online library.

García, F., \& Ilabaca, D. (2013). Ruptura de pareja, afrontamiento y bienestar psicológico en adultos jóvenes. Ajayu, 11(2). http://www.scielo.org.bo/scielo.php?script=sci_arttext\&pid=\$2077-21612013000200003

García, M., \& Romero, A. (2012). Mantenimiento en la relación de pareja: construcción y validación de dos escalas. Ridep, 1(34). https://docplayer.es/396539-Mantenimiento-en-la-relacion-de-parejaconstruccion-y-validacion-de-dos-escalas-relationship-maintenance-construction-and-validation-of-twoscales.html

Garrido, A., Reyes, A., Torres, L., \& Ortega, P. (2008). Importancia de las expectativas de pareja en la dinámica familiar. Enseñanza e Investigación en Psicología, 13(2), 231-238. https://www.redalyc.org/articulo.oa?id=29213203

Goodboy, A. K., Bolkan, S., Sharabi, L. L., Myers, S. A., \& Baker, J. P. (2020). The relational turbulence model: a meta-analytic review. Human Communication Research, 46(2-3), 222-249. https://doi.org/10.1093/hcr/hqaa002

Granillo, L., \& Sánchez, R. (2020). Deteriorando la salud en pareja. El papel de los eventos hirientes. Enseñanza e Investigación en Psicología, 2(2). https://revistacneip.org/index.php/cneip/article/view/107

Hair, J., Black, W., \& Anderson, R. (2010). Multivariate data analysis (7th Editio). Pearson Education.

Hawkins, A., Galovan, A., Harris, S., Allen, S., Allen, S., Roberts, K., \& Schramm, D. (2017). What are they thinking? A national study of stability and change in divorce ideation. Family Process, 56(4), 852-868. 
https://doi.org/10.1111/famp.12299

Hazan, C., \& Diamond, L. (2000). The place of attachment in human mating. Review of General Psychology, 4(2), 186-204. https://doi.org/10.1037/1089-2680.4.2.186

Hendrick, S. (1988). A generic measure of relationship satisfaction. Journal of Marriage and the Family, 50(1), 93. https://doi.org/10.2307/352430

Hernández, N., Alberti, P., Niñez, J., \& SAmaniego, M. (2011). Relaciones de género y satisfacción marital en comunidades rurales de Texcoco, Estado de México. Revista Internacional de Ciencias Sociales y Humanidades, 21(1), 39-64. https://www.redalyc.org/articulo.oa?id=65421407003

Holt, J., Smith, T., Baker, M., Harris, T., \& Stephenson, D. (2015). Loneliness and social isolation as risk factors for mortality. Perspectives on Psychological Science, 10(2), 227-237. https://doi.org/10.1177/1745691614568352

Hoy, A., Diiriye, A., \& Gunderson, E. (2022). Divorce ideation and "Deal breakers" among married gay men and lesbians: A qualitative exploration. Journal of Family Issues, 43(1), 164-185. https://doi.org/10.1177/0192513X21993854

Hu, L., \& Bentler, P. (1999). Cutoff criteria for fit indexes in covariance structure analysis: Conventional criteria versus new alternatives. Structural Equation Modeling: A Multidisciplinary Journal, 6(1), 1-55. https://doi.org/10.1080/10705519909540118

Huo, Y., \& Kong, F. (2014). Moderating effects of gender and loneliness on the relationship between self-esteem and life satisfaction in Chinese University students. Social Indicators Research, 118(1), 305-314. https://doi.org/10.1007/s11205-013-0404-x

Irani, E., Park, S., \& Hickman, R. (2021). Negative marital interaction, purpose in life, and depressive symptoms among middle-aged and older couples: evidence from the Health and Retirement Study. Aging \& Mental Health, 1-10. https://doi.org/10.1080/13607863.2021.1904831

Jong, J., Broese, M., Hoogendoorn, A., \& Smit, J. (2009). Quality of Marriages in Later Life and Emotional and Social Loneliness. The Journals of Gerontology Series B: Psychological Sciences and Social Sciences, 64B(4), 497-506. https://doi.org/10.1093/geronb/gbn043

Knoke, J., Burau, J., \& Roehrle, B. (2010). Attachment styles, Ioneliness, quality, and stability of marital relationships. Journal of Divorce \& Remarriage, 51(5), 310-325. https://doi.org/10.1080/10502551003652017 
Lawal, A., \& Okereke, C. (2021). Relationship satisfaction in cohabiting university students: evidence from the role of duration of cohabitation, Ioneliness and sex-life satisfaction. Vulnerable Children and Youth Studies, 16(2), 134-143. https://doi.org/10.1080/17450128.2020.1842574

Lee, S., Wickrama, K., Lee, T., \& O’Neal, C. (2021). Long-Term physical health consequences of financial and marital stress in middle-aged couples. Journal of Marriage and Family, 83(4), 1212-1226. https://doi.org/10.1111/jomf.12736

Lin, S., Li, J., \& Han, R. (2018). Coping humor of entrepreneurs: interaction between social culture and entrepreneurial experience. Frontiers in Psychology, 9. https://doi.org/10.3389/fpsyg.2018.01449

Lozano, L., García, E., \& Muñiz, J. (2008). Effect of the number of response categories on the reliability and validity of rating scales. Methodology, 4(2), 73-79. https://doi.org/10.1027/1614-2241.4.2.73

MacDonald, K., Kumar, A., \& Schermer, J. (2020). No laughing matter: How humor styles relate to feelings of Ioneliness and not mattering. Behavioral Sciences, 10(11), 165. https://doi.org/10.3390/bs10110165

Margelisch, K., Schneewind, K., Violette, J., \& Perrig, P. (2017). Marital stability, satisfaction and well-being in old age: variability and continuity in long-term continuously married older persons. Aging \& Mental Health, 21(4), 389-398. https://doi.org/10.1080/13607863.2015.1102197

Marilaf, M., San, M., Delgado, R., \& Vivanco, L. (2017). Empatía, soledad, desgaste y satisfacción personal en Enfermeras de cuidados paliativos y atención domiciliaria de Chile. Enfermería Clínica, 27(6), 379-386. https://doi.org/10.1016/j.enfcli.2017.04.007

Martin, R., \& Ford, H. (2018). The psychology of humor: An integrative approach (2da ed). Elsevier

Martin, R., \& Lefcourt, H. (1983). Sense of humor as a moderator of the relation between stressors and moods. Journal of Personality and Social Psychology, 45(6), 1313-1324. https://doi.org/10.1037/00223514.45.6.1313

Maté, C., \& Acarín, N. (2011). Encuesta sobre la elección de pareja a estudiantes de la Universitat Pompeu Fabra, Barcelona (20 a 27 años). Summa Psicológica, 8(1), 37-46. https://doi.org/10.18774/448x.2011.8.80

Mellor, D., Stokes, M., Firth, L., Hayashi, Y., \& Cummins, R. (2008). Need for belonging, relationship satisfaction, Ioneliness, and life satisfaction. Personality and Individual Differences, 45(3), 213-218. https://doi.org/10.1016/j.paid.2008.03.020

Minuchin, S. (1974). Familias y terapia familiar. Editorial Gedisa, S.A. 
Moral, J. (2008). Validación de la Escala de Valoración de la Relación en una muestra mexicana. REMA Revista electrónica de metodología aplicada, 13(1). https://doi.org/10.17811/rema.13.1.2008.1-12

Mund, M., \& Johnson, M. (2021). Lonely me, lonely you: loneliness and the longitudinal course of relationship satisfaction. Journal of Happiness Studies, 22(2), 575-597. https://doi.org/10.1007/s10902-020-00241-9

Mund, M., Weidmann, R., Wrzus, C., Johnson, M., Bühler, J., Burriss, R., Wünsche, J., \& Grob, A. (2020). Loneliness is associated with the subjective evaluation of but not daily dynamics in partner relationships. International Journal of Behavioral Development, 016502542095124. https://doi.org/10.1177/0165025420951246

Nezlek, J., \& Derks, P. (2001). Use of humor as a coping mechanism, psychological adjustment, and social interaction. Humor - International Journal of Humor Research, 14(4). https://doi.org/10.1515/humr.2001.011

Núñez, C., Ramos, C., Serpa, A., \& Oluwaseun, 0. (2021). Adaptation of the personality type inventory based on enneagram in Peruvian university students of health sciences. Journal of Research in Medical and Dental Science, 9(5). https://www.jrmds.in/articles/adaptation-of-the-personality-type-inventory-basedon-enneagram-in-peruvian-university-students-of-health-sciences-77092.html

Olapegba, P., Chovwen, C., Ayandele, 0., \& Ramos, C. (2021). Fear of COVID-19 and preventive health behavior: mediating role of post-traumatic stress symptomology and psychological distress. International Journal of Mental Health and Addiction. https://doi.org/10.1007/s11469-021-00557-4

Oliva, A., Pertegal, M., Antolín, L., Reina, M., Ríos, M., Hernando, Á., Parra, Á., Pascual, D., \& Estévez, R. (2011). Desarrollo positivo adolescente y los activos que lo promueven: Un estudio en centros docentes andaluces. Universidad de Sevilla. Departamento de Psicología Evolutiva y de la Educación. https://idus.us.es/handle/11441/32695

Ramírez, M., \& Lee, S. (2012). Factores asociados a la satisfacción vital en adultos mayores de 60 años. Polis (Santiago), 11(33), 407-428. https://doi.org/10.4067/S0718-65682012000300020

Ramos, C., Dávila, A., Castañeda, J., Ramírez, Y., \& Serpa, A. (2021). Estructura e invarianza factorial de una escala de perfeccionismo infantil adaptada para adolescentes. Revista de Psicopatología y Psicología Clínica, 26(2), 141-154. https://doi.org/10.5944/rppc.27301

Ramos, C., \& Sáenz, J. (2021). Estructura y análisis diferencial del ítem de acorde a sexo y edad del Test de Inteligencia General Matrices en estudiantes universitarios. Revista de Investigación en Psicología, 24(1), 73-90. https://doi.org/10.15381/rinvp.v24i1.20616 
Rodríguez, E., Veray, J., \& González, J. (2019). Sexualidad, satisfacción en la pareja y satisfacción con la vida en una muestra de matrimonios cristianos de Puerto Rico. Revista Salud y Conducta Humana, 5(1), 99112.

https://static1.squarespace.com/static/50c39c53e4b097533b3492dd/t/5ca75cd68165f53ef60c2199/1554 472152471/Articulo+Rodriguez-Robledo+2018+\%287\%29.pdf

Saporta, N., Scheele, D., Lieberz, J., Stuhr, F., Hurlemann, R., \& Shamay, S. (2021). Opposing association of situational and chronic loneliness with interpersonal distance. Brain Sciences, 11(9), 1135. https://doi.org/10.3390/brainsci11091135

Saroglou, V., Lacour, C., \& Demeure, M.-E. (2010). Bad humor, bad marriage: humor styles in divorced and married couples. Europe's Journal of Psychology, 6(3), 94-121. https://doi.org/10.5964/ejop.v6i3.210

Schumacker, R. E., \& Lomax, R. G. (1996). A Beginner's Guide to Structural Equation Modeling (2nd Editio). Psychology Press. https://doi.org/10.4324/9781410610904

Sellin, N., \& Keeves, J. (1997). Path analysis with latent variables. In J. P. Keeves (Ed.), Educational research, methodology, and measurement: An international handbook. Oxford: Pergamon Press.

Tian, Y., Zhang, S., Wu, R., Wang, P., Gao, F., \& Chen, Y. (2018). Association between specific internet activities and life satisfaction: The mediating effects of loneliness and depression. Frontiers in Psychology, 9. https://doi.org/10.3389/fpsyg.2018.01181

Turan, N., Durgun, H., Kaya, H., Aştı, T., Yilmaz, Y., Gündüz, G., Kuvan, D., \& Ertaş, G. (2020). Relationship between nursing students' levels of internet addiction, loneliness, and life satisfaction. Perspectives in Psychiatric Care, 12474. https://doi.org/10.1111/ppc.12474

Urbano, A., Iglesias, M., \& Martínez, R. (2019). Satisfacción general y sexual con la relación de pareja en función del género / General and Sexual Satisfaction with the Couple Relationship According to the Gender. Revista Española de Investigaciones Sociológicas. https://doi.org/10.5477/cis/reis.165.143

Urbano, A., Martínez, R., \& Iglesias, M. (2018). Parenthood as a determining factor of satisfaction in couple relationships. Journal of Child and Family Studies, 27(5), 1492-1501. https://doi.org/10.1007/s10826017-0990-3

Urbano, A., Martínez, R., \& Iglesias, M. (2021). Revisión de la investigación sobre relaciones de pareja en países hispanohablantes (2000-2018). Interdisciplinaria Revista de Psicología y Ciencias Afines, 38(3), 25-48. https://doi.org/10.16888/interd.2021.38.3.2

Valdez, J., González, N., \& Sánchez, Z. (2005). Elección de pareja en universitarios mexicanos. Enseñanza e 
Investigación en Psicología, 10(2), 355-367. http://ri.uaemex.mx/handle/20.500.11799/38864

Vela, L., Booth, M., Wanzer, M., \& Vallade, J. (2013). Relationships among humor, coping, relationship stress, and satisfaction in dating relationships: replication and extension. Communication Research Reports, 30(1), 68-75. https://doi.org/10.1080/08824096.2012.746224

Vidal, L., Rivera, S., Díaz, R., \& Méndez, I. (2012). Elaboración de una escala de permanencia en la relación de pareja. Revista Iberoamericana de Diagnóstico y Evaluación - e Avaliação Psicológica, 1(33), 199-225. https://www.redalyc.org/articulo.oa?id=459645437011

Ward, P., Lundberg, N., Zabriskie, R., \& Berrett, K. (2009). Measuring marital satisfaction: A comparison of the revised dyadic adjustment scale and the satisfaction with married life scale. Marriage \& Family Review, 45(4), 412-429. https://doi.org/10.1080/01494920902828219

Wickrama, K. A. S., \& O'Neal, C. W. (2021). Midlife marital and financial stress and the progression of later-life health problems for husbands and wives. Journal of Aging and Health, 33(9), 685-697. https://doi.org/10.1177/08982643211004364

Wickrama, K., Lee, T., \& O'Neal, C. (2020). Marital strain trajectories over a quarter century and spouses' Ioneliness: Couple-level and individual pathways. Journal of Social and Personal Relationships, 37(3), 821-842. https://doi.org/10.1177/0265407519879512

Wongpakaran, N., Wongpakaran, T., Pinyopornpanish, M., Simcharoen, S., Suradom, C., Varnado, P., \& Kuntawong, P. (2020). Development and validation of a 6-item Revised UCLA Loneliness Scale (RULS-6) using Rasch analysis. British Journal of Health Psychology, 25(2), 233-256. https://doi.org/10.1111/bjhp.12404

Zicavo, N., \& Vera, C. (2011). Incidencia del ajuste diádico y sentido del humor en la satisfacción marital. Revista De Psicología (Trujillo), 12(1), 74-89. https://revistas.ucv.edu.pe/index.php/revpsi/article/view/670 